\title{
ON THE METHOD OF SUCCESSIVE APPROXIMA- TIONS FOR VOLTERRA INTEGRAL EQUATIONS
}

\author{
SEPPO HEIKKILÄ
}

\section{Introduction}

Walter has in [4] proved an existence and uniqueness theorem for Volterra integral equations of the form

$$
x(t)=y(t)+\int_{0}^{t} k(t, s, x(s)) d s
$$

in a Banach space, by the method of successive approximations (see also $[3])$.

In this paper we shall prove an analogous theorem with more general hypotheses, and derive estimates for the solution of (1), with minimal solutions of the scalar comparison equation

$$
u(t)=v(t)+\int_{0}^{t} \omega(t, s, u(s)) d s
$$

as estimate functions.

The results so obtained are then applied to the initial value problem

$$
x^{\prime}(t)=y^{\prime}(t)+f(t, x(t)), \quad x(0)=y(0),
$$

in a Banach space, with the scalar comparison problem

$$
u^{\prime}(t)=v^{\prime}(t)+g(t, u(t)), \quad u(0)=v(0) .
$$

\section{Notations}

Let $X$ be a Banach space with norm $\|\cdot\|$. Given $T \cdot>0$, denote $J=$ $[0, T]$, and let $C(J)$ and $C_{+}(J)$ be the spaces of all continuous mappings from $J$ into $X$ and into the nonnegative reals $\mathbf{R}_{+}$, respectively, with the topology of uniform convergence. For $y \in C(J)$ define $|y| \in C_{+}(J)$ by 


$$
|y|(t)=\|y(t)\|, \quad t \in J
$$

and for $u, v \in C_{+}(J)$

$$
u \leqq v \text { if and only if } u(t) \leqq v(t) \text { for each } t \in J
$$

Any constant mapping of $C(J)$ or of $C_{+}(J)$ is denoted by its value.

Denote by $\mathcal{K}_{0}^{+}$the class of all functions $\omega$ from the set $\{(t, s, r) \in J \times$ $\left.J \times \mathbf{R}_{+} \mid s \leqq t\right\}$ into $\mathbf{R}_{+}$for which $\omega(t, s, r)$ is measurable in $s \in[0, t]$ for each $(t, r) \in J \times \mathbf{R}_{+}$, continuous in $(t, r) \in J \times \mathbf{R}_{+}$for almost every $s \in[0, t]$, and for each $M>0$ there is an integrable function $h: J \rightarrow \mathbf{R}_{+}$such that

$$
\omega(t, s, r) \leqq h(s)
$$

for $0 \leqq s \leqq t \leqq T$ and $0 \leqq r \leqq M$. Correspondingly, denote by $\mathcal{K}_{0}$ the class of all mappings $k$ from $\{(t, s, z) \in J \times J \times X \mid s \leqq t\}$ into $X$ for which $k(t, s, z)$ is strongly measurable in $s \in[0, t]$ for each $(t, z) \in J \times X$, continuous in $(t, z) \in J \times X$ for almost every $s \in[0, t]$, and for each $M>0$ there is an integrable $h: J \rightarrow \mathbf{R}_{+}$such that

$$
\|k(t, s, z)\| \leqq h(s)
$$

whenever $0 \leqq s \leqq t \leqq T$ and $\|z\| \leqq M$.

Applying the Dominated Convergence Theorem for Lebesgue integrals one can show that the integral

$$
\Omega u(t)=\int_{0}^{t} \omega(t, s, u(s)) d s
$$

exists in the Lebesgue sense for $\omega \in \mathcal{K}_{0}^{+}, u \in C_{+}(J)$ and $t \in J$, and that (2.3) defines a mapping $\Omega: C_{+}(J) \rightarrow C_{+}(J)$. Respective properties of Bochner integrals ensure that for $k \in \mathcal{K}_{0}$,

$$
K x(t)=\int_{0}^{t} k(t, s, x(s)) d s, \quad t \in J, \quad x \in C(J),
$$

defines a mapping $K: C(J) \rightarrow C(J)$.

Via the definitions (2.3) and (2.4) the integral equations (1) and (2) may be written in the forms

$$
\begin{aligned}
& x=y+K x, \\
& u=v+\Omega u,
\end{aligned}
$$

respectively.

\section{Existence and uniqueness theorems}

Making minor modifications to the proof of Theorem I.7.XII in [4] we obtain 
Theorem 3.1. Assume that $k \in \mathcal{K}_{0}$, and for $0 \leqq s \leqq t \leqq T, z, \bar{z} \in X$,

$$
\|k(t, s, z)-k(t, s, \bar{z})\| \leqq \omega(t, s,\|z-\bar{z}\|),
$$

where $\omega \in \mathcal{K}_{0}^{+}$such that

(i) $\omega(t, s, r)$ is nondecreasing in $r \in \mathbf{R}_{+}$for $0 \leqq s \leqq t \leqq T$;

(ii) the mapping $\Omega$, defined by (2.3), has $u=0$ as the only fixed point;

(iii) for each $C>0$ there is $w \in C_{+}(J)$ satisfying

$$
w \geqq C+\Omega w .
$$

Then for $y, x_{1} \in C(J)$ the successive approximations

$$
x_{n+1}=y+K x_{n}, \quad n \in \mathbf{N}=1,2, \ldots,
$$

with $K$ given by (2.4), converge on $J$ uniformly to a unique solution of (1).

Proof. From (i) it follows that $\Omega$ is nondecreasing, and from (3.1) that

$$
|K x-K \bar{x}| \leqq \Omega(|x-\bar{x}|), \quad x, \bar{x} \in C(J),
$$

whence

$$
\left|x_{n+1}-x_{1}\right| \leqq\left|y-x_{1}\right|+\left|K x_{1}\right|+\Omega\left(\left|x_{n}-x_{1}\right|\right), \quad n \in \mathbf{N} .
$$

These properties, together with (iii), imply by induction that

$$
\left|x_{n}-x_{1}\right| \leqq w, \quad n \in \mathbf{N},
$$

for any $w \in C_{+}(J)$, satisfying (3.2) with

$$
C \geqq\left|y-x_{1}\right|+\left|K x_{1}\right| .
$$

From (3.3), (3.4) and (3.5) it follows by induction that

$$
\left|x_{n+m}-x_{m}\right| \leqq u_{m}, \quad n, m \in \mathbf{N},
$$

where the functions $u_{m}$ are defined by

$$
u_{1}=w, u_{m+1}=\Omega u_{m}, \quad m \in \mathbf{N} .
$$

The sequence $\left(u_{m}\right)$ is nonincreasing and nonnegative, whence by (ii) it can be shown to converge to 0 -function (see the proof of Theorem I.7.XII in [4]), uniformly on $J$. From (3.6) it then follows that $\left(x_{n}\right)$ convegges in $C(J)$. The conditions given for $\mathcal{K}_{0}$, together with the Dominated Convergence Theorem for Bochner integrals, ensure that the limit mapping of $\left(x_{n}\right)$ is a solution of (1).

The uniqueness can be proved as in Theorem I.7.XII of [4].

Denote by $\mathcal{K}$ the class of those $k \in \mathcal{K}_{0}$ for which (2.2) holds for some integrable $h: J \rightarrow \mathbf{R}_{+}$and for all $(t, s, z) \in J \times J \times X, s \leqq t$. 
Corollary 3.1. Let $k \in \mathcal{K}$ satisfy (3.1) with $\omega \in \mathcal{K}_{0}^{+}$such that the hypotheses (i) and (ii) of Theorem 3.1 hold. Then (1) has for each $y \in C(J)$ a unique solution on $J$.

Proof. Define

$$
\bar{\omega}(t, s, r)=\min \{\omega(t, s, r), 2 h(s)\}, \quad 0 \leqq s \leqq t \leqq T, \quad r \geqq 0,
$$

where $h$ is the majorant of $k$ in (2.2). Then $\bar{\omega} \in \mathcal{K}_{0}^{+},(3.1)$ holds with $\omega$ replaced by $\bar{\omega}$, and $\bar{\omega}$ satisfies the hypotheses (i) and (ii) of Theorem 3.1. Also (iii) holds, because

$$
w(t)=C+\int_{0}^{t} 2 h(s) d s
$$

satisfies (3.2). Thus the assertion follows from Theorem 3.1.

Remark 3.1. Corollary 3.1 simplifies the Existence and Convergence Theorem I.7.XII of [4] in the sense that the hypothesis

$(\gamma)$ for each $C>0$ there is $\varrho \in C_{+}(J)$ satisfying

$$
\varrho \geqq C \text { and } \varrho \geqq \Omega \varrho,
$$

of the Theorem is not specified in the Corollary. In Theorem 3.1 we use the stronger condition (iii) in place of $(\gamma)$, also to show the boundedness of $\left(x_{n}\right)$ (see (3.5)), whence we may assume that $k \in \mathcal{K}_{0}$, instead of $k \in \mathcal{K}$. Furthermore, (iii) (but not $(\gamma)$ ) is sufficient for the considerations of the next section.

An essential point where the method of Walter, used in the proof for the uniform convergence of $\left(x_{n}\right)$, differs from other methods (see for ex. [2]) is that the equicontinuity of $\left(x_{n}\right)$ is not needed.

\section{Integral inequalities}

The estimates derived in the present section for solutions of (1) are based on

Lem ma 4.1. Let $\omega \in \mathcal{K}_{0}^{+}$satisfy the hypotheses (i) and (iii) of Theorem 3.1. Then given $v \in C_{+}(J)$ the equation

$$
u=v+\Omega u,
$$

with $\Omega$ given by (2.3), has the minimal solution $u$ on $J$. If $\left(y_{n}\right)$ is a convergent sequence in $C(J)$ such that

$$
\left|y_{1}\right| \leqq v \text { and }\left|y_{n+1}\right| \leqq v+\Omega\left(\left|y_{n}\right|\right), \quad n \in \mathbf{N},
$$

then the limit mapping $\tilde{y}$ of $\left(y_{n}\right)$ satisfies

$$
|\tilde{y}| \leqq u
$$


Proof. From (i) it follows that $\Omega$ is nondecreasing, whence the sequence $\left(u_{n}\right)$ defined by

$$
u_{1}=v, \quad u_{n+1}=v+\Omega u_{n}, \quad n \in \mathbf{N},
$$

is nondecreasing and bounded above by any $w$ satisfying (3.2) with $C \geqq|v|$. Since $\left(u_{n}\right)$ is also equicontinuous (cf. the proof of Theorem I.2.II in [4]), it converges uniformly on $J$. The continuity of $\omega(t, s, r)$ in $r$, together with the Dominated Convergence Theorem, implies that the limit $u$ of $\left(u_{n}\right)$ is a solution of $\left(2^{\prime}\right)$. The solution $u$ is minimal, since

$$
u_{n} \leqq \bar{u}, \quad n \in N
$$

for any solution $\bar{u}$ of $\left(2^{\prime}\right)$.

The last conclusion of lemma is a consequence of

$$
\left|y_{n}\right| \leqq u_{n}, \quad n \in \mathbf{N},
$$

which follows from (4.1) and (4.3) by induction.

Theorem 4.1. Let $k$ and $\omega$ satisfy the hypotheses of Theorem 3.1, and let $x$ be the solution of (1) with a given $y \in C(J)$. Assume further that $z \in C(J)$ and $v \in C_{+}(J)$ satisfy

$$
|z-y-K z| \leqq v
$$

Then

$$
|z-x| \leqq u
$$

where $u$ is the minimal solution of $\left(2^{\prime}\right)$.

Proof. Let $\left(x_{n}\right)$ be the sequence of successive approximations, with $x_{1}=y+K z$ as the first approximation, converging to $x$. Then it is easy to see that

$$
\left|z-x_{1}\right| \leqq v \text { and }\left|z-x_{n+1}\right| \leqq v+\Omega\left(\left|z-x_{n}\right|\right), \quad n \in \mathbf{N}
$$

Thus (4.1) holds for $y_{n}=z-x_{n}$, which by Lemma 4.1 implies the assertion.

Corollary 4.1. With the hypotheses of Theorem 3.1,

$$
|x-y| \leqq u,
$$

where $u$ is the minimal solution of $\left(2^{\prime}\right)$ with $v=|K y|$. If $\bar{x}$ is the solution of (1) with $y$ replaced by another mapping $\bar{y}$ from $C(J)$, then

$$
|\bar{x}-x| \leqq u,
$$

where $u$ is the minimal solution of $\left(2^{\prime}\right)$ with $v=|\bar{y}-y|$.

Proof. Choose first $z=y$ and then $z=\bar{x}$ in Theorem 4.1 .

As another consequence of Theorem 3.1. and Lemma 4.1 we obtain 
Theorem 4.2. Assume that $k$ satisfies the hypotheses of Theorem 3.1, and that for $(t, s, z) \in J \times J \times X, s \leqq t$,

$$
\|k(t, s, z)\| \leqq \bar{\omega}(t, s,\|z\|),
$$

where $\bar{\omega} \in \mathcal{K}_{0}^{+}$such that the hypotheses (i) and (iii) of Theorem 3.1 hold for $\omega=\bar{\omega}$. Then the solution of (1) with a given $y \in C(J)$ satisfies

$$
|x| \leqq \bar{u},
$$

where $\bar{u}$ is the minimal solution of

$$
\bar{u}=|y|+\bar{\Omega} \bar{u}
$$

with

$$
\bar{\Omega} \bar{u}(t)=\int_{0}^{t} \bar{\omega}(t, s, \bar{u}(s)) d s, \quad t \in J, \quad \bar{u} \in C_{+}(J)
$$

Proof. Let $\left(x_{n}\right)$ be the sequence of the successive approximations given by (3.3) with $x_{1}=y$. Then (4.1) holds for $y_{n}=x_{n}, v=|y|$ and $\Omega=\bar{\Omega}$, so that (4.8) follows from (4.2).

The first conclusion of Corollary 4.1 yields the following local version of Theorem 3.1:

Corollary 4.2. Let $B$ be an open subset of $X$, and let $k:\{(t, s, z) \in J \times$ $J \times B \mid s \leqq \dot{t}\} \rightarrow X$ satisfy the hypotheses of Theorem 3.1 for all $(t, s, z)$, $(t, s, \bar{z}) \in J \times J \times B, s \leqq t$. Then for each continuous mapping $y: J \rightarrow B$, the integral equation (1) has a unique solution on $\left[0, T_{1}\right)$ with

$$
T_{1}=\sup \left\{t \in J \mid u(s) \leqq d\left(y(s), B^{c}\right) \text { for } 0 \leqq s \leqq t\right\}
$$

where $u$ is the minimal solution of $\left(2^{\prime}\right)$ with $v=|K y|$, and $d\left(y(t), B^{c}\right)$ denotes the distance between $y(t)$ and the complement $B^{c}$ of $B$ in $X$.

This corollary shows an advantage of minimal solutions of $\left(2^{\prime}\right)$ as estimating functions, compared to corresponding maximal ones, obtained by other methods (see for ex. [4], I.4.I).

\section{Consequences for differential equations}

Denote by $A C_{+}(J)$ the class of all absolutely continuous functions $u$ : $J \rightarrow \mathbf{R}_{+}$, and by $A C(J)$ the class of those $u \in C(J)$ which are strongly。differentiable almost everywhere on $J$, and for which $|u| \in A C_{+}(J)$. Let $\mathcal{F}_{0}$ denote the class of such mappings of $\mathcal{K}_{0}$ which do not depend on $t$, and $\mathcal{F}_{0}^{+}$the respective subclass of $\mathcal{K}_{0}^{+}$.

From the properties of Bochner integrals (see [1] Chapter 3) it follows that for each $y \in A C(J)$ the formula 


$$
y(t)=y(0)+\int_{0}^{t} y^{\prime}(s) d s
$$

holds on $J$, and that the mapping $t \rightarrow \int_{0}^{t} f(s, x(s)) d s, t \in J$, belongs to $A C(J)$ whenever $f \in \mathcal{F}_{0}$ and $x \in C(J)$. Analogous properties hold trivially in the scalar case. These facts imply that the initial value problem

$$
x^{\prime}(t)=y^{\prime}(t)+f(t, x(t)), \quad x(0)=y(0),
$$

with $y \in A C(J)$ and $f \in \mathcal{F}_{0}$, is representable in the form

$$
x(t)=y(t)+\int_{0}^{t} f(s, x(s)) d \mathrm{~s},
$$

and similarly,

$$
u^{\prime}(t)=v^{\prime}(t)+g(t, u(t)), \quad u(0)=v(0),
$$

with $v \in A C_{+}(J)$ and $g \in \mathcal{F}_{0}^{+}$, in the form

$$
u(t)=v(t)+\int_{0}^{t} g(s, u(s)) d s
$$

Thus the results of Sections 3 and 4 are applicable for (3). From Theorem 3.1 we have

Theorem 5.1. Assume $f \in \mathcal{F}_{0}$ and for $(s, z),(s, \bar{z}) \in J \times X$

$$
\|f(s, z)-f(s, \bar{z})\| \leqq g(s,\|z-\bar{z}\|),
$$

where $g \in \mathcal{F}_{0}^{+}$satisfying

(i) for each $s \in J, g(s, r)$ is nondecreasing in $r \in \mathbf{R}_{+}$;

(ii) $u(t) \equiv 0$ is the maximal solution of

$$
u^{\prime}(t)=g(t, u(t)), \quad u(0)=0
$$

(iii) for each $C>0$ there is $w \in C_{+}(J)$ for which

$$
w(t) \geqq C+\int_{0}^{t} g(s, w(s)) d s, \quad t \in J .
$$

Then given $y \in A C(J)$, (3) has a unique solution $x$ (a. e.) on $J$, and $x$ can be obtained as the uniform limit of successive approximations with any mapping of $C(J)$ as the first approximation.

The results of Section 4 and formula (5.1) yield

Theorem 5.2. Let $f$ and $g$ satisfy the hypotheses of Theorem 5.1, and let $x(t)=x\left(t, y^{\prime}(t), y(0)\right)$ denote the solution of $(3)$, and $u(t)=u\left(t, v^{\prime}(t), v(0)\right)$ the minimal solution of (4). If $z \in A C(J)$ and $v \in A C_{+}(J)$ satisfy 


$$
\left\|z(t)-y(t)-\int_{0}^{t} f(s, z(s)) d s\right\| \leqq v(t), \quad t \in J
$$

then

$$
\|z(t)-x(t)\| \leqq u(t), \quad t \in J
$$

In particular,

and

$$
\|y(t)-x(t)\| \leqq u(t,\|f(t, y(t))\|, 0)
$$

$$
\|y(0)-x(t)\| \leqq u\left(t,\left\|y^{\prime}(t)+f(t, y(0))\right\|, 0\right) .
$$

If $\bar{x}(t)=x\left(t, \bar{y}^{\prime}(t), \bar{y}(0)\right)$ is another solution of $(3)$, then

and

$$
\|\bar{x}(t)-x(t)\| \leqq u\left(t,\left\|\bar{y}^{\prime}(t)-y^{\prime}(t)\right\|,\|\bar{y}(0)-y(0)\|\right)
$$

$$
\|\bar{x}(t)-x(t)-\bar{y}(0)+y(0)\| \leqq u\left(t,\left\|\bar{y}^{\prime}(t)-y^{\prime}(t)\right\|, 0\right) .
$$

If there is $\bar{g} \in \mathcal{F}_{0}^{+}$satisfying the hypotheses (i) and (iii) imposed on $g$ in Theorem 5.1 , and if

then

$$
\|f(t, z)\| \leqq \bar{g}(t,\|z\|), \quad(s, z) \in J \times X,
$$

$$
\|x(t)\| \leqq \bar{u}(t), \quad t \in J,
$$

where $\bar{u}$ is the minimal solution of

$$
\bar{u}^{\prime}(t)=\left\|y^{\prime}(t)\right\|+\bar{g}(t, \bar{u}(t)), \quad \bar{u}(0)=\|y(0)\| .
$$

Examples 5.1. The Osgood function

$$
g(t, r)=p(t) \psi(r), \quad(t, r) \in J \times \mathbf{R}_{+}
$$

where $p: J \rightarrow \mathbf{R}_{+}$is integrable and $\psi: \mathbf{R}_{+} \rightarrow \mathbf{R}_{+}$a continuous and nondecreasing function for which the integrals $\int_{0}^{1} d r / \psi(r)$ and $\int_{i}^{\infty} d r / \psi(r)$ diverge, belongs to $\mathcal{F}_{0}^{+}$and satisfies the conditions (i) and (ii) of Theorem 5.1. Also (iii) holds with any $w$ satisfying

$$
\int_{C}^{w(t)} \frac{d r}{\psi(r)} \geqq \int_{0}^{t} p(s) d s, \quad t \in J .
$$

To get an example of (4) with nonunique solutions, define

$$
\begin{aligned}
& g(t, r)=r \quad \text { for } 0 \leqq r<e^{-t}, \quad t \in J ; \\
& g(t, r)=e^{-t} \quad \text { for } e^{-t} \leqq r<3-2 e^{-t}, \quad t \in J ; \\
& g(t, r)=e^{-t}+\left(r-3+2 e^{-t}\right)^{\frac{1}{2}} \quad \text { for } r \geqq 3-2 e^{-t}, \quad t \in J .
\end{aligned}
$$

this $g$ belongs to $\mathcal{F}_{0}^{+}$and satisfies the hypotheses (i)-(iii) of Theorem 5.1, and the initial value problem 


$$
u^{\prime}(t)=e^{-t}+g(t, u(t)), \quad u(0)=1
$$

has

$$
u_{*}(t)=3-2 e^{-t} \text { and } u^{*}(t)=3-2 e^{-t}+\frac{t^{2}}{4}
$$

as the minimal and maximal solutions, respectively.

Remark 5.1. The closed interval $J=[0, T]$ can be replaced in above considerations by $\left[t_{0}, T\right),-\infty<t_{0}<T \leqq \infty$, the convergence of the successive approximations being uniform on every compact subset of $\left[t_{0}, T\right)$. In case $T=\infty$, the example above shows that the minimal solutions of (3.2), which are as estimators in (4.5)-(4.8), may be bounded, whereas the corresponding maximal solutions may be unbounded.

\section{References}

[1] Hille, E., and R. S. Phillips: Functional analysis and semi-groups. - Amer. Math. Soc. Colloquium Publications Vol. 31, Providence, R. I., 1957.

[2] Vidossich, G.: Global Convergence of Successive Approximations. - J. Math. Anal. Appl. 45, 1974, 285-292.

[3] Walter, W.: Über sukzessive Approximation bei Volterra-Integralgleichungen in" mehreren Veränderlichen. - Ann. Acad. Sci. Fenn. Ser. A I 345, 1965, $1-32$.

[4] - - Differential and Integral Inequalities. - Ergebnisse der Mathematik und ihrer Grenzgebiete 55, Springer-Verlag, Berlin - Heidelberg - New York, 1970.

University of Oulu

Department of Mathematics

SF-90100 Oulu 10

Finland

Received 19 September 1974 